\title{
Evaluating SPARQL Query on Semantic Data Store in Cloud Platform
}

\author{
Ankit Kulkarni, Mayur Sagavkar, Anupriya Elumalai*,N.Ch.S.N Iyengar \\ School of Computing Science and Engineering, VIT University \\ *Department of Information Technology, Ibri College of Technology, Oman
}

\begin{abstract}
Cloud computing provides virtualization of services, has enabled opportunities for integration and collaboration of many e-businesses around world. Also, semantic web technology has been evolving in parallel, which uses RDF and OWL as storage formats for business data. Integration of semantic web technology on cloud platform provides an efficient way of managing data for e-commerce based applications. Many databases storage approaches have been proposed for e-commerce applications. However, there is a need to model the data storage based on semantic information so that the access to data store based on semantic behavior of user context and past history. The proposed work models a mobile phone e-commerce application which uses RDF data store which is maintained in cloud environment. SPARQL queries are used for access and retrieval mechanism.
\end{abstract}

Keywords: E-business, Semantic Web, RDF, SPARQL, Cloud

\section{Introduction}

Cloud computing is one of the most important research area as well as most adopted technology in the recent time. Database is also moved into the cloud which is provided as feature of cloud as software as a service also called as Database as a service. Cloud provides huge storage area and the data can be stored as distributed manner which provide performance. We can use storage as service provided by many of cloud service providers like amazon web service and can store the database over the cloud and can use the service any time as require. In today's research work semantic web is one of the most important research areas. Semantic web already acting as an attractive package to next generation web based model .Highly unstructured data available on the web based source put forwards challenges to search engine present today. One can even look the semantic as part of optimization. One of the major component associated the semantic structure is Resource Description Framework (RDF) commonly used to define each object available on web uniquely and in structured manner. This paper represents the intersection of semantic web and cloud computing. As Semantic web is a one of the major research area which deals with semantic heterogeneity and semantic integration. Semantic web gives us the idea of how one can represents the data uniquely over the internet with the help of $\mathrm{RDF}$ and ontology language. Semantic web solves the problem of semantic heterogeneity and provide the integration of cross domain data. Whereas cloud computing is also one of the major research field.

This paper provides the integration of sematic web with cloud services. Integration of cloud services and semantic web provide a good functionality for e-commerce, e-banking, e-business area. This paper implements e-commerce application to provide functionality and provide a better way of representing the database. The data which is available on internet is in a very huge amount and the biggest problem with this data is that it is not standardized data i.e. data is not semantically or structurally similar. This is because of the industries producing the data individually according to their own requirement and there is 
not any standard to make this data similar in nature. So there is a need of creating such standards to make this data similar so that one can easily integrate this data, semantic web deals with the same problem.

There are some of the issues that need to be getting solved in order to implement the work carried out in this paper. Following are some of the research challenges should be faced in order to deal with semantic web services.

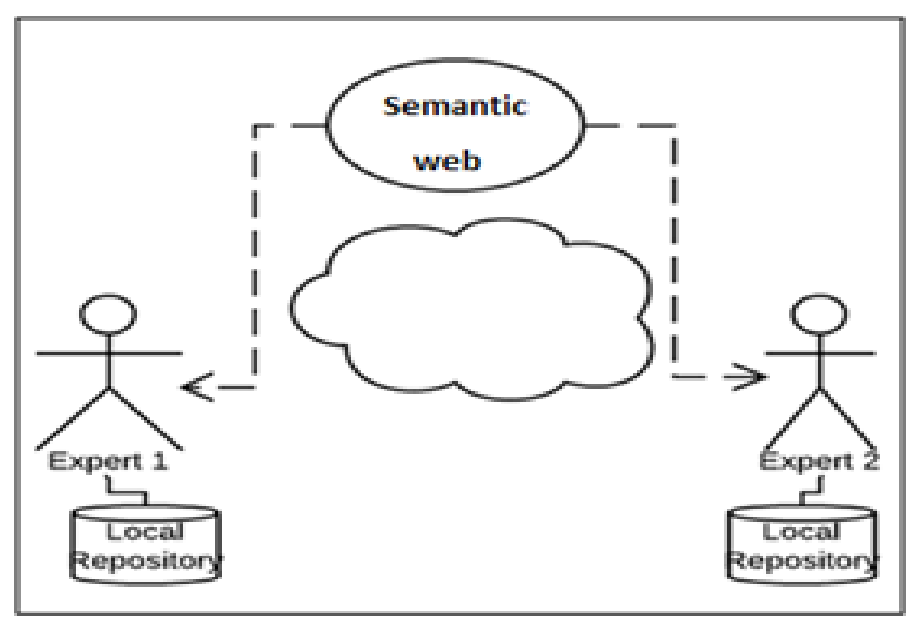

Figure 1. Semantic Web Integration for Cross-domain Repositories

\subsection{Semantic Heterogeneity}

The major part of the data generated over the internet has been produced by the industries. These industries are creating their data using their own standards and also this data is not machine understandable data i.e. machine cannot recognize this data as knowledge and cannot process this data.so to make this data machine understandable so that even machines can process the data and can be enabled.

\subsection{Semantic Integration}

Semantic integration is a solution of semantic heterogeneity though providing semantic heterogeneity itself is a major task it requires additional effort like implementing standards or transformation of underlying concepts of tools and languages.

Semantic integration solution for distributed and semantically heterogeneous data makes it easy to share domain independent distributed data. Fig.1 shows the representation of semantic web which provide semantic integration. Semantic integration enables the matching of schemas, identifying the duplicate the duplicate entries reconciliation of inconsistencies. In semantic integration we study about the mapping of vocabularies between two different data sources. Following are the two major issues we need to study when considered under semantic integration.

How to connect two systems that uses different platforms, protocols so they can exchange information? How to translate data between two different systems uses different terminology for the same concept? The answer to such questions can be given by analyzing and finding a particular way of mapping between to heterogeneous system so that these systems can efficiently communicate the meaningful data. The communication between such systems can be event based or request/response model based. 


\section{Surviving Techniques}

[12] proposed the implementation of trust model for providing reliability of data on internet. The data on internet is not reliable there is no source which can perform the authentication of the data. There is a need of providing reliability of data so that one can have trust on the data. This paper uses propagation method which provides the trust for the user of the data. It uses both centralized and distributed method to provide the trust of the data. [8] proposed the way of implementing the virtualize services over the cloud. It is one of the research challenge and a lot of research is already been carried out in this area. This can be useful when different organization wants to integrate their different services into a single service for providing functionality. It uses a cloud registry to access the registry entry and from there user can access the data it provides a great flexibility for user as it is available on cloud.

[16] proposed the next generation search engines will implements data as semantic web data. These search engine use drilldown approach to organize the data. Using this approach user can search the data by using some specific key words and it will provide a better way of searching the data. This approach gives recommendation systems as the implementation method. It helps the user to provide better search and navigational methods. Currently only user can understand the content of the data not the machine but integration of web semantic concepts will allow machine to understand and process the data. It will provide the reasoning of data and creation of knowledge base for machine and machine can process the data easily. Francesca Bugiotti et al proposed RDF data management over the cloud architecture. This paper proposed architecture for distributed RDF data over the amazon cloud services. This paper uses RDF data format to store the data and SPARQL to query the data in a distributed manner. This approach used the data to be distributed over the cloud in machine understandable format and the query is also provided in distributed manner.

In today world scenario users are using the web interface in order to access the data. So there is a need of integrated access of services is, desired by the user in order to make it more efficient. By using the web portals user not only want to access the information but also various services provided by the portals. But in traditionally integration of web services is not possible. Suppose that there are two web services one which provide the information about a movie theatres in the city and second which provide the booking facility. If a user wants to see available theaters in the city by using first web service and to book a ticket by using second one so this will give a better association of the service. It is possible to span the query across the data but to integrate the services is somehow difficult. To achieve this goal there is a need of a platform independent high level web portal integrated architecture which will provide the way of dealing with nonheterogeneous data.

XML is one of the platform independent data interchange standard on the web. XML carries the data in the form of the tree structure, but two semantically equivalent documents can have different structures the two XML may have the same information but different structure. For merging the data from two semantically same but having different structure XML documents there is a need to implement the semantic integration. Yvette E. Geologo et al described in his paper the database management as a cloud service where data can be distributed over the cloud and sharing of resources, softwares can be carried out because of which growing interest in outsourcing database and database management activities can be seen in recent years. The implementation of the database over cloud provide the users a better way of accessing the data on demand.

[17] proposed the challenges occurred when dealing with semantic web over cloud environment. Semantic web provide of understanding the data by machine as compare to html pages but on the other hand the performance of accessing this new format of data should be efficient so that performance will not deteriorate. This paper deals with the 
problem of design such a trival system. This paper deals with the data managing concets deals with the huge storage of RDF triplets and how one can manage such huge storage efficiently using different aspects of query spawning and distributed query processing. Arindem khaled et al proposed a token based access control mechanism for RDF. This paper describe the access mechanism for multiple access of data entity in a cloud environment and It describe the mechanism of Access token tuples based on Access Token, Element, Element Type and Element Name. The access type can be categories based on different subtypes such as Data access, Predicate access, Object access etc. The token assignment is done based on access token assignment and based on defined time stamp.

Table 1 shows the Drawbacks of Semantic Integration Surveyed techniques. Table 2 shows the challenges in the existing methodology with cloud databases. The challenges can be resolved by semantic cloud data stores such as RDF and OWL which are maintained be semantic cloud web services.

Table 1. Drawbacks of Semantic Integration Surveyed Techniques

\begin{tabular}{|c|l|l|}
\hline S.No & \multicolumn{1}{|c|}{ Surviving techniques } & \multicolumn{1}{c|}{ Drawbacks/obsolete } \\
\hline 1 & $\begin{array}{l}{[1] \text { has proposed a knowledge base }} \\
\text { framework to perform transformation of } \\
\text { data models of software engineering } \\
\text { tools }\end{array}$ & $\begin{array}{l}\text { Better way of exchanging data } \\
\text { between cross domain business entitie } \\
\text { ban implemented. But the } \\
\text { integration is not for heterogeneous } \\
\text { data stores. }\end{array}$ \\
\hline 2 & $\begin{array}{l}{[2] \text { has proposed an ontology based }} \\
\text { framework for XML semantic } \\
\text { integration }\end{array}$ & $\begin{array}{l}\text { XML schema integration is possible } \\
\text { and XML queries can be spawned over } \\
\text { multiple XML schemas locally. But } \\
\text { the integration is not for heterogeneous } \\
\text { data stores. }\end{array}$ \\
\hline 3 & $\begin{array}{l}{[3] \text { has proposed Semantic based web }} \\
\text { services integration in P2P }\end{array}$ & $\begin{array}{l}\text { User can use to different web services } \\
\text { in integrated manner. But the } \\
\text { integration is not for heterogeneous } \\
\text { data stores. }\end{array}$ \\
\hline 4 & $\begin{array}{l}{[4] \text { has proposed an Approach to }} \\
\text { Ontology-Based Semantic Integration } \\
\text { for PLM Object }\end{array}$ & $\begin{array}{l}\text { Performing mapping between only } \\
\text { PLM object and ontology object based } \\
\text { on ontology based model (protégé) }\end{array}$ \\
\hline 5 & $\begin{array}{l}{[5] \text { has proposed Integration of }} \\
\text { heterogeneous } \\
\text { environments for the automation } \\
\text { systems lifecycle }\end{array}$ & $\begin{array}{l}\text { Simple aggregation of the components, } \\
\text { improved coordination between the } \\
\text { tools. However, integration is not for } \\
\text { heterogeneous data stores. }\end{array}$ \\
\hline 6 & $\begin{array}{l}{[6] \text { has proposed Semantic-integration }} \\
\text { research in the database community }\end{array}$ & $\begin{array}{l}\text { Broad way description of Data } \\
\text { translation and matching does not } \\
\text { handle heterogeneous data stores } \\
\text { integration. }\end{array}$ \\
\hline 7 & $\begin{array}{l}\text { [7] has proposed Automating Cloud } \\
\text { Services Life cycle through Semantic } \\
\text { The Framework uses uses SPARQL } \\
\text { query and RDF format for query and } \\
\text { accessing of data. However, } \\
\text { integration is not for heterogeneous } \\
\text { data stores }\end{array}$ \\
\hline
\end{tabular}


Table 2. Challenges in the Existing Methodology with Cloud Databases

\begin{tabular}{|c|l|l|}
\hline S.No & \multicolumn{1}{|c|}{ Challenge } & \multicolumn{1}{|c|}{ Description And Related Issues } \\
\hline 1 & Scalability & $\begin{array}{l}\text { One of the main features of Cloud is scalability which } \\
\text { implies that resources can be scaled-up or scaled-down } \\
\text { dynamically without causing any interruption in the } \\
\text { service. It puts challenges on developers to develop } \\
\text { databases such that they can support unlimited number of } \\
\text { concurrent users and data growth. }\end{array}$ \\
\hline 2 & $\begin{array}{l}\text { High availability and } \\
\text { Fault Tolerance }\end{array}$ & $\begin{array}{l}\text { Availability of database implies that database is up and } \\
\text { running. It becomes necessary to replicate data across } \\
\text { large geographic distances to provide high data } \\
\text { availability, durability and high levels of fault tolerance. }\end{array}$ \\
\hline 3 & $\begin{array}{l}\text { Heterogeneous } \\
\text { Environment }\end{array}$ & $\begin{array}{l}\text { Users want to access diverse applications from different } \\
\text { locations and devices such as mobiles, tablets, notepads } \\
\text { and computers. Since user applications and data } \\
\text { structured or unstructured) vary in nature, it becomes } \\
\text { difficult to predefine how users will use the system. }\end{array}$ \\
\hline 4 & $\begin{array}{l}\text { Data Consistency and } \\
\text { Integrity }\end{array}$ & $\begin{array}{l}\text { Data integrity is the most critical requirement of all } \\
\text { business applications and is maintained through database } \\
\text { constraints. The lack of data integrity results in } \\
\text { unexpected outputs and it becomes difficult to maintain } \\
\text { the consistency. }\end{array}$ \\
\hline 5 & $\begin{array}{l}\text { Simplified } \\
\text { Interface }\end{array}$ & $\begin{array}{l}\text { Cloud Database is distributed. Querying distributed } \\
\text { database is a major challenge that cloud developers face. } \\
\text { A distributed query has to access multiple nodes of cloud } \\
\text { database. There should be a simplified and standardized } \\
\text { query interface for querying the database. }\end{array}$ \\
\hline 6 & $\begin{array}{l}\text { Interoperability and } \\
\text { Data physically stored in a particular country, is subject } \\
\text { to Privacy rules and regulations of that country. If data is } \\
\text { encrypted using a key not located at the host, then it is } \\
\text { little safer. }\end{array}$ \\
$\begin{array}{l}\text { Data Portability is the ability to run components written } \\
\text { for one cloud provider in another cloud provider's } \\
\text { environment. }\end{array}$ \\
\hline 7
\end{tabular}

\section{Cloud Computing and Semantic Web Fundamentals}

Cloud Computing has been extensively adopted in various application due to this elasticity, scalability, and fault tolerance. Web service is one of the recent evolutionary topics. It has brought heterogeneous applications, platforms, remote services, etc. together. The semantic feature of web service gives us an advantage of context-aware; QoS-aware; behavior-aware web service discovery mechanism.

Semantic Computing bridges and integrates various domains such as software engineering, natural language processing, artificial intelligence, grid computing, pervasive computing, etc. As a result semantic maintenance and processing of data that correspond to real time application become easy. Therefore, there is a need to maintain and process semantic data for real time applications in cloud environment. Figure 2 shows the evolution of data format whereas Figure 3 shows the functional block diagram of web service discovery mechanism in cloud environment. 


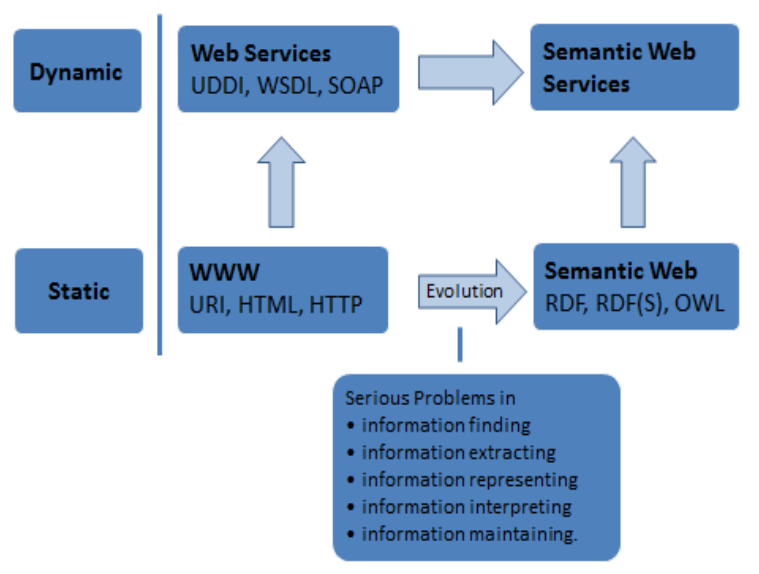

Figure 2. Evolution of Data Storage to Semantic Web Specific Data

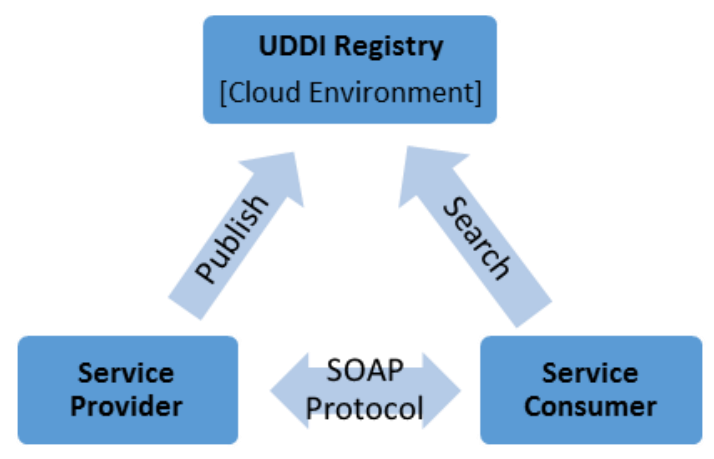

Figure 3. Web Service Discovery Mechanism in Cloud Environment

\section{Proposed Methodology}

Semantic web service is any service that is available over the Internet, uses a standardized XML messaging system, and is not tied to any one operating system or programming language. This can be discoverable using service registries.

The working steps of web service discovery mechanism in cloud environment are as follows.

Begin

Step 1: The service provider implements the service and makes it available on the Internet in cloud environment.

Step 2: This is a logically centralized directory of services maintained in cloud environment. It therefore serves as a centralized storage for companies and their services.

Step 3: This is any consumer of the web service. The requestor utilizes an existing web service by opening a network connection and sending an XML request message. 
Step 4: XML request is semantically matched in the UDDI registry and an XML reply message is created containing the description of the matched entries of the respective web services

Step 5: Cloud Server send back the XML reply message on the same network to web service requestor.

Step 6: web service requestor extracts the location of the desired web service and invokes it maintained over cloud environment

End

\section{Proposed Architecture}

Figure 4 shows the proposed architecture for cloud based semantic integration of cross domain resources.

\subsection{Non Cross domain Data Sources}

In the proposed architecture the lower layer represents non cross domain data sources. These sources are having different data models and tools and their own standards and structure. These resources query in their local query language and get the result in the local standard format across the cross domain data sources.

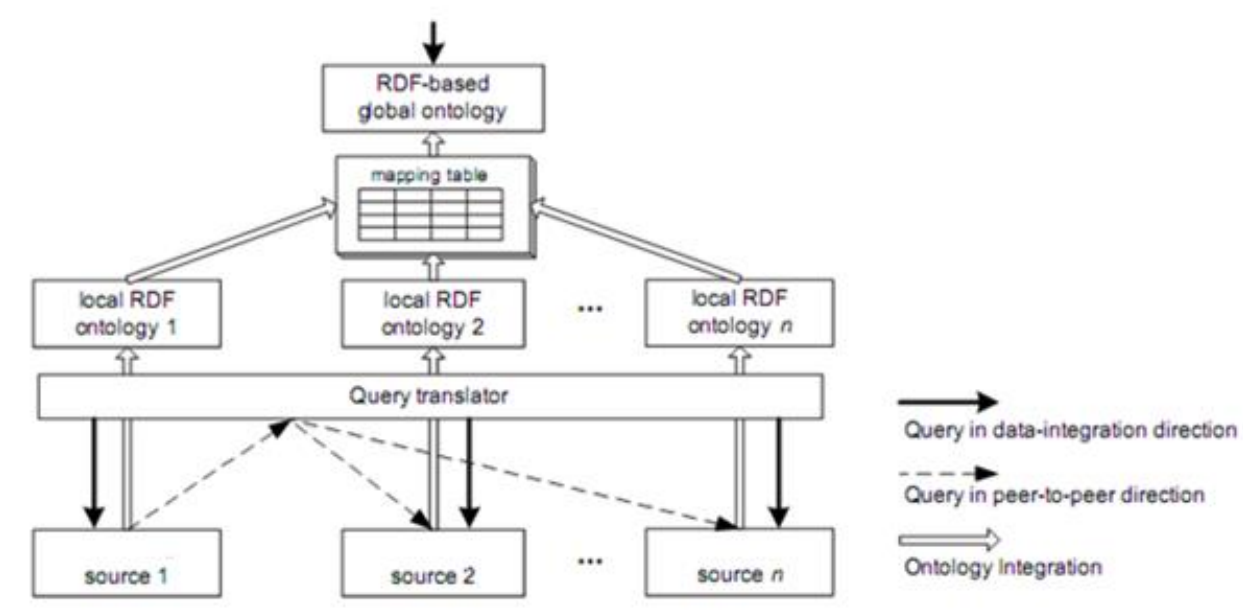

Figure 4. Semantic Integration Framework for Cross Domain Data Store Integration 


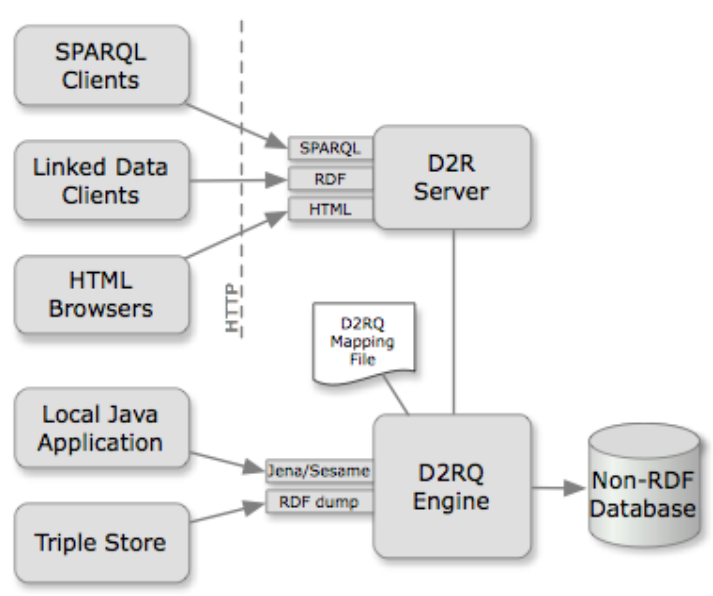

Figure 5. D2RQ Server Architecture

\subsection{Query Translator}

Query translator is used to transform the query from one standard to another. It acts as an intermediate between different sources and RDF mapping table. This paper illustrates D2RQ server as a query translator which translates the query from local data standards to RDF based query standard.

The D2RQ platform is used for accessing the relational databases as RDF graphs. It provides the RDF based access to relational database. By using D2RQ server one can perform functionalities like query a non-RDF database based on SPARQL queries, Linked data access, create custom dumps of the database in RDF formats for loading into an RDF store. D2RQ Platform consists of the following modules.

1. The D2RQ Mapping Language, a declarative mapping language for describing the relation between ontology and a relational data model.

2. The D2RQ Engine, a plug-in for the Jena Semantic Web toolkit, which uses the mappings to rewrite Jena API calls to SQL queries against the database and passes query results up to the higher layers of the frameworks.

3. The D2R Server, an HTTP server that provides a Linked Data view, a HTML view for debugging and a SPARQL Protocol endpoint over the database.

\subsection{The RDF mapping table.}

There are two steps related to the ontology integration process viz Schema transformation and Ontology merging. In the first method we use RDFL (Resource Description Framework Schema) to model each XML schema as a local RDF ontology for a uniform representation. The main aim of such method is to preserve the structure of the XML schema. In the second step we merge the local schema to generate the global ontology. We can also introduce the additional domain specific knowledge. One mapping table also introduces which contain the mapping between the local and the global ontology. We consider the mapping of two XML schema carried out we assume that these two XML schema are equivalent because in some cases they may not be semantically equivalent. There are two roles of a global ontology. One it provides the user access to the data with a uniform query interface, and second it serves as a mediation mechanism for accessing the distributed data through any of XML sources. As shown in the fig 4 the translation of the two queries can occur in two ways. 
1. Data integration: The system translates the RDF (Directed to global ontology) query into multiple sub-queries (One for each XML sources).

2. Peer to Peer integration: In this type of query processing technique the query proposed by a user against the XML schema is propagated to all the mediated distributed sources.

The proposed solution should give us solution for query access in real time system. While the users of these systems still see a single schema (whether relational or $\mathrm{XML}$ ), queries are translated on the fly to appropriate queries over the individual data sources, and results are combined appropriately from partial results obtained from the sources. Consequently, answers returned to the user are always based on fresh data. In any data-sharing architecture, reconciling semantic heterogeneity is the key. No matter whether the query is issued on the fly, or data are loaded into a warehouse, or whether data are shared through web services or in a peer-to-peer fashion, the semantic differences between data sources need to be reconciled. Typically, these differences are reconciled by semantic mappings. These are expressions that specify how to translate data from one data source into another in a way that preserves the semantics of the data or, alternatively, reformulate a query posed on one source into a query on another source. The working steps of evaluating semantic data store in cloud platform are shown below.

Begin

Step 1: User request for services through the device user interface with the query request details. The details are sent in platform specific message structure.

Step 2: This request is handled through semantic web service for access and retrieval from RDF data store [SaaS].

Step 3: Query details are then passed to SPARQL Query Evaluation Engine which evaluates the query and prepares the result set

Step 4: The result set is then passed to semantic web service in XML format

Step 5: The Web service forwards the query result set to the end user.

Step 6: End User application process the XML and displays the Query Outcome.

End

\section{Implementation}

\subsection{Resource Description Format [RDF] Example}

Following RDF file is designed for Book schema based using foaf ontology using which one can represent book information in machine understandable format.

<rdf:RDF xmlns:rdf=http://www.w3.org/1999/02/22-rdf-syntax-ns\#

xmlns:rdfs $=$ http://www.w3.org/2000/01/rdf-schema\# xmlns: foaf=http://xmlns.com/foaf/0.1/>

<foaf:Book rdf:ID="Data Stucure" $>$

$<$ foaf:author >GS Baluja</foaf:author>

$<$ foaf:eddition $>$ http://www.htc.com</foaf:eddition>

$<$ foaf:price >Desire L</foaf:price >

$<$ foaf:OS $>$ Punit Malhotra </foaf:OS>

$<$ foaf:publication >ANDROID</foaf: publication > 


\author{
<foaf:year>2hrs 25min</foaf:year> \\ $\langle$ foaf:issn $>$ BOLLYWOOD</foaf:issn $>$ \\ $<$ foaf:rating >Social</foaf:rating > \\ $</$ foaf:Book $>$ \\ $\langle/ r d f: R D F\rangle$
}

\title{
6.2 Sample SPARQL Query Evaluation
}

As per the user choice the requested company, Company name will be passed on to the web server through HttpRequest using live internet.

After getting this name at server side, SPARQL query will be executed. This SPARQL query executing using JENA Framework which is using RDF file as a database. The output of query executing will passed on to the android user in the form of XML.

\section{SELECT ?o}

\section{WHERE}

\section{\{?s foaf: name?o.\}}

In this implementation Netbeans for server side and Eclipse and client side IDE. Figure 6 shows mobile Company list and Figure 7 shows specification of particular company.

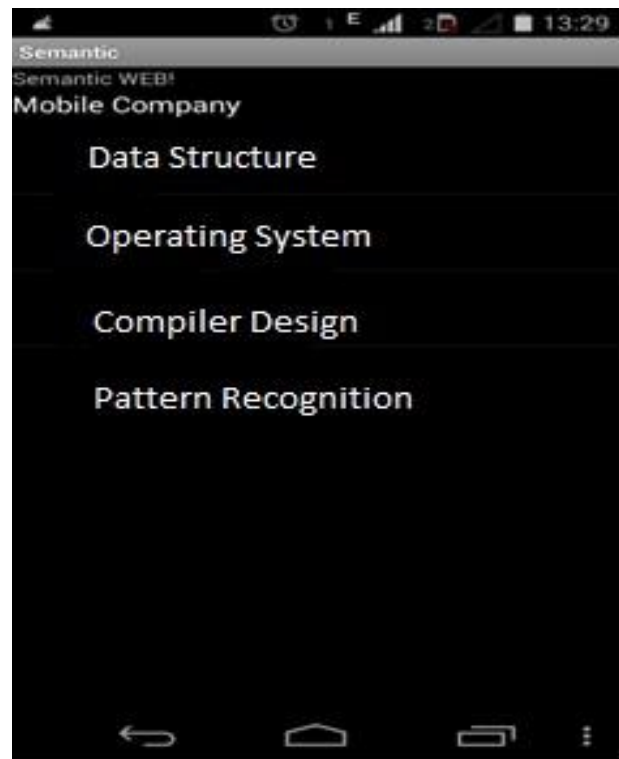

Figure 6. Mobile Company List

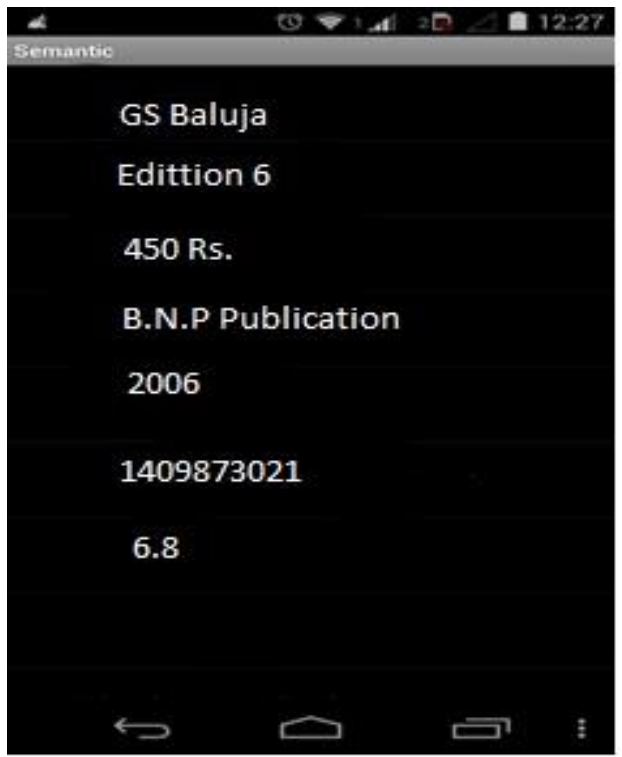

Figure 7. Specification of Company

\section{Integrating Structure and Semantics: Case Study on Book Ontology}

To support the reversible query translation process between the structural query (XQuery) and the semantic query (RDQL), we choose to extend the vocabulary of RDF to make it capable of representing not only the semantics but also the structure of the data.

Figure 8 shows the RDF ontologies $\left(\mathrm{S1}^{\prime}\right.$ and $\left.\mathrm{S} 2^{\prime}\right)$ that result respectively from the transformation of S1 and S2. 


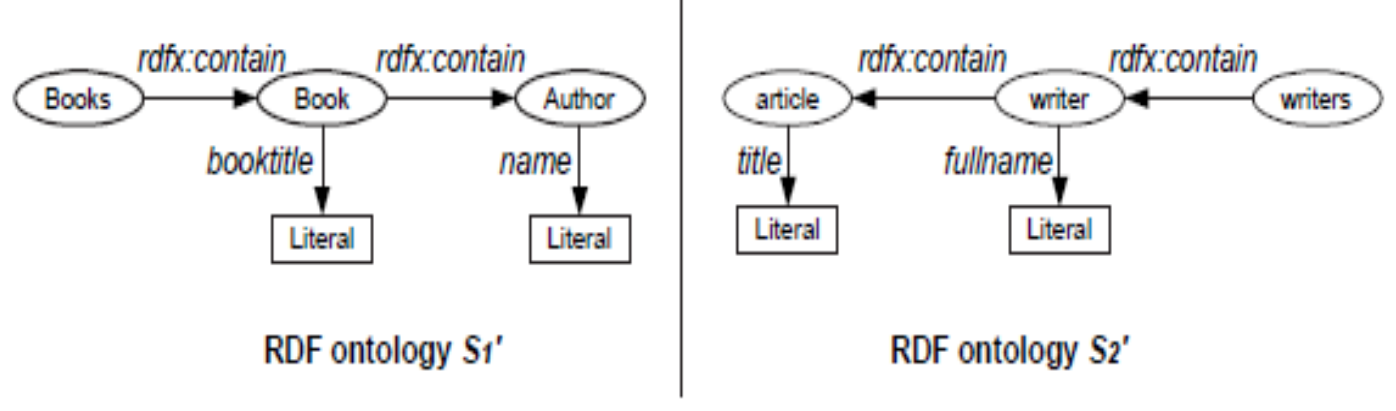

Figure 8. Global Ontology Merged from Local Ontologies

\subsection{Element-level Transformation}

The element-level transformation defines the basic classes and properties of the local RDF ontology according to the transformation correspondences shown in Table 3, with the structural relationships between the elements not being considered for the time being.

\section{Table 3. Element Level Transformation}

\begin{tabular}{|l|l|}
\hline XML Schema concepts & RDF Schema Concepts \\
\hline Attribute & Property \\
\hline Simple-type element & Property \\
\hline Complex-type element & Class \\
\hline
\end{tabular}

\subsection{Structure-level Transformation}

The structure-level transformation encodes the hierarchical structures of the XML schema into the local RDF ontology. Table 4 lists the mapping information between the XML source $\mathrm{S} 1$ and the local RDF ontology $\mathrm{S} 1^{\prime}$.

Table 4. Mapping between S1 and S1/

\begin{tabular}{|l|l|}
\hline XPath expressions in S1 & RDF expressions in S1' \\
\hline /books & Books \\
\hline /books/book & Book \\
\hline /books/book/@booktitle & Book.booktitle \\
\hline /books/book/author & Author \\
\hline /books/book/author/@name & Author.name \\
\hline
\end{tabular}

\subsection{Merge Mechanism of Local Ontologies into Global Ontologies}

The ontology that results from merging two local RDF ontologies in the case study is shown in Figure 9 


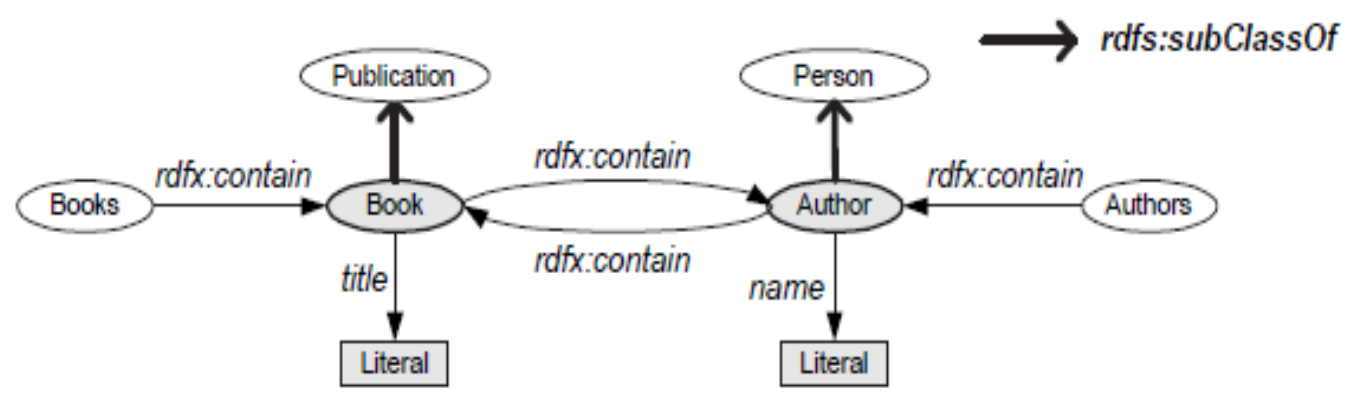

Figure 9. Global Ontology Merged from Local Ontologies

The grayed classes and properties are merged classes and properties from the original ontologies. For instance, the class Book is merged from Book in $\mathrm{S}^{\prime}$ and Article in S2'.

\subsection{Mapping Table between the Global Ontology and Local RDF Ontologies}

The mapping table contains the mapping information between the local RDF ontologies and the global RDF ontology. In general, if a class, property, or relationship between classes $\mathrm{p}$ in the global ontology is the result of merging pi and pj from different local ontologies, then a mapping in the form of (p, pi, pj ) is generated. Table 5 lists all the mappings in the book ontology case study.

Table 5. Mapping Table of Book Ontology

\begin{tabular}{|l|l|l|}
\hline $\begin{array}{l}\text { RDF expressions in } \\
\text { the global ontology }\end{array}$ & RDF expressions in S1 & RDF Expression in S2' \\
\hline Books & Books & - \\
\hline Book & Book & Article \\
\hline Book.title & Book.booktitle & Article.title \\
\hline Authors & - & Writers \\
\hline
\end{tabular}

\section{Conclusion}

The kind of data available on web is in unstructured manner. Basic semantic web gives framework to store and use the data in structured manner which in much more efficient than current scenario of web. The data stored in semantic form for ex if RDF is kind of data that is machine understandable form .SPARQL query is one of the powerful retrieval patterns in semantic web. This proposed work gives basic idea to ensure the working of semantic web on one of the famous android based platform. The gives rise to idea that it can also be applicable to cross platform devices also.

\section{References}

[1] T. Moser and S. Biffl, "Semantic Integration of Software and Systems Engineering Environments", Part C: Applications and Reviews, IEEE Transactions on Systems, Man, and Cybernetics, vol. 42, no. 1, (2012), pp: 38 - 50

[2] I. F. Cruz, H. Y. Xiao and F. H. Hsu, "An ontology-based framework for XML semantic integration", International Database Engineering and Applications Symposium, (2004).

[3] J. Z. Li and B. Xu, "SEWSIP: semantic based Web services integration in P2P", IEEE International Workshop on Service-Oriented System Engineering, (2005).

[4] J. A. Kwak and H. S. Yong, "An Approach to Ontology-Based Semantic Integration for PLM Object", IEEE International Workshop on Semantic Computing and Applications, (2008). 
[5] S. Biffl, A. Schatten and A. Zoitl, "Integration of heterogeneous engineering environments for the automation systems lifecycle", 7th IEEE International Conference on Industrial Informatics, Publication Year, (2009).

[6] A. H. Doan and A. Y. Halevy, "Semantic-integration research in the database community", AI Magazine - Special issue on semantic integration, vol. 26, no. 1, (2005), pp. 83-94

[7] K. P. Joshi, Y. Yesha and T. Finin, "Automating Cloud Services Life cycle through Semantic", IEEE Transactions on Services Computing, vol. 7, no. 1, (2014), pp. $109-122$.

[8] E. P. Wach, "A hurastic as a basis for an adaptive e-commerce recommender system", 12th International Conference on Intelligent Systems Design and Applications (ISDA), (2012).

[9] V. Vinothina, R. Sridaran and P. Ganpathi, "A Survey on Resource Allocation Strategies in cloud computing", International Journal of Advanced Computer Science and Application, vol. 3, no. 6, (2012), pp. $97-104$

[10] C. Spawar and R. Bwagh, "Priority based dynamic resource allocation in cloud computing Cloud and services", International Symposium on Cloud and Services Computing, (2012).

[11] M. Katebi and S. D. Katebai, "Trust model analysis for the semantic web", Second International Conference on development in e-system Engineering. Abu Dhabi, UAE, (2009).

[12] M. Klems, S. Tai, L. Sharwtz and G. Grabarnik, "Automating the Delivery of IT service Continuity Management through cloud Service Orchestration", IEEE/IFIP Network Operations and Management Symposium - NOMS: Mini-Conference, (2010).

[13] T. Eimuri and S. Salehi, "Investgating the use of semantic-based website to Improve Recommendation Quality", Second International Conference on Computer Research and Development, (2010); Kuala Lumpur, Malaysia.

[14] J. Kirschnick, J. M. A. Calero, L. Wilcock and N. Edwards, "Toward an Architecture for the Autamated provisioning of cloud services", IEEE Communications Magazine, (2010).

[15] A. M. Canthadai, "Shopping search and semantic web", IEEE Consumer Communication and Network Conference, (2011); Las Vegas, NV.

[16] M. F. Husain, "Data intensive query processing for large RDF graphs using cloud computing tools", IEEE 3rd International Conference on cloud computing", CLOUD, (2010); Miami, FL.

[17] A. Khaled, "A token based access control system for RDF data in the clouds", 2nd IEEE International Conference on Cloud Computing Technology and Science, CloudCom., Indiana University, (2010).
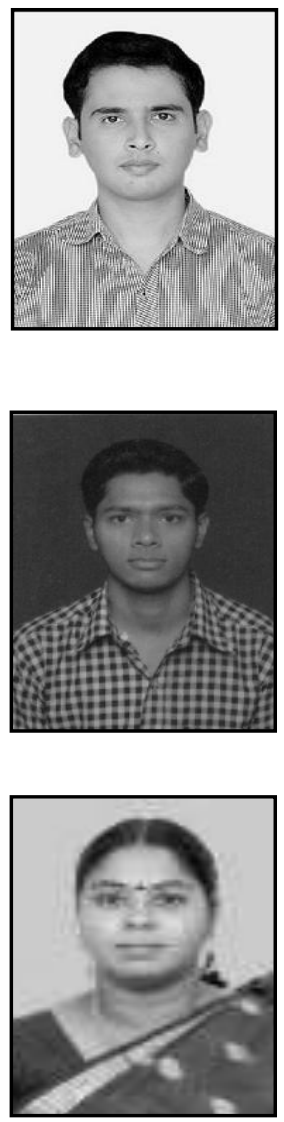

\section{Authors}

Ankit kulkarni, (b 1991), he is currently pursuing M.Tech in Computer Science and Engineering for VIT University Vellore632014, Tamilnadu, India. He has completed his graduation in Computer Science and Engineering from RGPV University Madhya Pradesh India. His research interests include Sematic Web, Parallel Programming, Cloud Computing, etc.

Mayur Sagavkar, (b 1989), he is pursing M.Tech with School of Computing Science and Engineering, VIT University, Vellore632014, India. He received his B. Tech in Computer Science Engineering from PVG's College of Engineering and Technology, Pune, Maharashtra, India. His research interests include Sematic Web, Image Processing, Cloud Computing, etc.

Anupriya Elumalai, she received her B.E (CSE) Madras University in 1997, both M.Tech.(C SE) Ph.D from VIT University in 2004 \& 2014.. Currently she is faculty at Information Technology, Ibri College of Technology, Sultanate of Oman. Her research interests include Peer-to-Peer Data Management, Data Mining, Knowledge discovery from text data and Information 
Retrieval. She has 15 publications in Journals and Conferences to her credit. She is a member of ISTE, AIENG and IEEE.

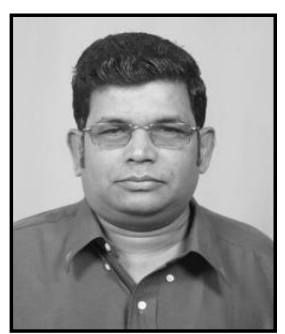

N.Ch.S.N. Iyengar, (b 1961), he is currently Senior Professor at the School of Computing Science and Engineering at VIT University, Vellore-632014, Tamil Nadu, India His research interests include Agent-Based Distributed secure Computing, Intelligent Computing, Network Security, Secured Cloud Computing and Fluid Mechanics. He has authored several textbooks and had nearly 167 research publications in reputed peer reviewed international journals. He served as PCM/reviewer for many international and IEEE conferences. He is Editor in Chief for International Journal of Software Engineering and Applications( IJSEA) of AIRCC, Guest Editor for Special Issue on Cloud Computing and Services of Int'l J. of Communications, Network and System Sciences and Editorial Board member for International Journals like IJConvC (Inderscience -China), IJCA (USA) etc. 